\title{
The Evaluation and Followup of Children Referred to Pediatric Endocrinologists for Short Stature
}

\author{
David Wyatt, ${ }^{1}$ Katrina L. Parker, ${ }^{2}$ Stephen F. Kemp, ${ }^{3}$ Jane Chiang, ${ }^{4}$ and D. Aaron Davis ${ }^{5}$ \\ ${ }^{1}$ Medical College of Wisconsin, 9000 West Wisconsin Avenue, MC C520, P.O. Box 1997, Milwaukee, WI 53201-1997, USA \\ ${ }^{2}$ Morehouse School of Medicine, 720 Westview Drive South West, Atlanta, GA 30310-1495, USA \\ ${ }^{3}$ University of Arkansas for Medical Sciences, Arkansas Children's Hospital 1 Children's Way, 4301 West Markham Street, Little Rock, \\ AR 72205, USA \\ ${ }^{4}$ Genentech, Inc., Stanford University Medical Center, 1 DNA Way, South San Francisco, CA 94080, USA \\ ${ }^{5}$ Medical Science Liaison, Genentech, Inc., 1 DNA Way, South San Francisco, CA 94080, USA
}

Correspondence should be addressed to David Wyatt, dwyatt@mcw.edu

Received 22 December 2009; Revised 30 March 2010; Accepted 22 April 2010

Academic Editor: Michael Bernd Ranke

Copyright (C) 2010 David Wyatt et al. This is an open access article distributed under the Creative Commons Attribution License, which permits unrestricted use, distribution, and reproduction in any medium, provided the original work is properly cited.

\begin{abstract}
Objective. To characterize the pediatric endocrinologists' evaluation and followup of short-statured patients. Study Design. Observational study of 21,548 short-statured children (April 1996 to December 1999). Baseline demographics, laboratory testing, height standard deviation score (SDS), target height, and height relative to target height were analyzed at initial and return visits with the specialist. Patients were scheduled for at least one return visit and no recombinant human growth hormone therapy was administered. Results. Mean patient age was 8.6 years with a mean height SDS of -2.1 . Patients were predominantly male (69\%), prepubertal (73\%), and white (76\%). Few screening tests were obtained during initial evaluation. Nearly $40 \%$ of children did not return for their second scheduled visit. The follow-up rate was unrelated to demographics or degree of short stature. Conclusions. Low return rates limit specialists' ability to monitor growth or obtain laboratory testing over time. Further studies are needed to determine which tests should be obtained at the initial clinic visit as well as the basis for the low return rate in this group of children.
\end{abstract}

\section{Introduction}

Growth can be used as a proxy for a child's health, nutrition, and psychosocial well being. In the United States, approximately 2.2 million children under 18 years of age have heights below the third percentile [1]. Most will be healthy, but a minority will have a pathologic etiology for their short stature. Among the short-statured children who are referred to a pediatric endocrinologist for the evaluation of growth failure, only a minority are treated with recombinant human growth hormone (rhGH). The clinical characteristics of those who are evaluated for growth failure but do not receive rhGH treatment are unknown.

When first seeing a short-statured referral, the pediatric endocrinologist must determine which patient requires prompt extensive evaluation versus more conservative monitoring. This decision is based on a combination of history, genetic growth potential, prior laboratory testing, and the degree of growth failure observed. Unfortunately, the available growth records at the time of referral can often be incomplete [2]. Forward monitoring of the child's untreated growth rate could be a valuable part of the evaluation. However, the percentage of children who return for such monitoring and the extent of laboratory screening eventually performed are unknown.

The diagnostic and therapeutic approach to a patient with short stature may differ among pediatric endocrinologists. In 1995, a written survey was distributed to pediatric endocrinologists in an attempt to determine the degree of variability in the evaluation of the short child [3]. The survey asked the 251 respondents (413 surveys distributed; 61\% response rate) to identify which screening tests were most commonly used to aid in the diagnosis of growth failure and to obtain feedback about recommendations for follow-up 
care. The survey found that the majority of endocrinologists stated that they were using a similar approach, especially in regards to laboratory assessment. However, it is not known whether this consensus would be seen with a more direct monitoring of actual clinical practice.

The goal of this study was to characterize the initial diagnostic assessment and follow-up of untreated short-statured subjects referred to pediatric endocrinologists. These results were then compared with the findings of the physician survey from 1995. Substudy 8 was specifically designed within the National Cooperative Growth Study (NCGS) to collect enrollment demographics as well as clinical and laboratory data from patients referred to pediatric endocrinologists for growth failure but not treated with rhGH. A preliminary overview of the NCGS Substudy 8 data was previously described [4]. The detailed analysis is presented in this paper.

\section{Patient Selection and Methods}

The NCGS is an ongoing postmarketing surveillance study of children receiving Genentech rhGH. Approximately 400 institutions from across the United States contribute to this long-running registry. The NCGS Substudy 8 was a prospective, observational study designed to characterize the enrollment demographics of subjects referred to NCGS investigators for evaluation of growth failure and to delineate the assessment process and diagnostic procedures used. Participating centers were invited to enroll patients in Substudy 8 from April 1996 to December 1999. The substudy was closed to further data collection in December 2001. Data were collected from pediatric endocrinologists as they examined short or poorly growing children referred by their primary health care provider. After the initial evaluation for short stature, investigators were instructed to enroll all consenting patients who had significant growth failure worthy of monitoring over more than one follow-up visit. Patients without significant growth failure who did not require follow-up visits were excluded from the study. Each participating pediatric endocrinologist subjectively defined significant growth failure. Patients did not receive rhGH before or during the observation period. If rhGH therapy was initiated during observation, the patient was excluded. All patients enrolled in Substudy 8 whose age at referral was 20 years or younger and whose height standard deviation score (SDS) could be calculated were included in these analyses. Where appropriate, institutional review board approval and informed consent for transmission of anonymized data were obtained.

Baseline data including sex, race/ethnic origin, birth weight and length, biological parents' heights, age at pubertal onset, referral source, and medical history were collected. At baseline and subsequent visits, investigators recorded the date of the visit, height, weight, Tanner stage, current medications, radiologic tests obtained (including bone age and central nervous system imaging), laboratory assessments (including evaluation of the growth hormone-insulin-like growth factor [GH-IGF] axis), presumed etiology of short stature (if known), and treatment plan.
To estimate a child's target height, his/her gender, the parents' heights, and a regression-to-the-mean factor of 0.8 described by Tanner [5] were used, where the adjusted target height formulae are as follows.

Male adjusted target height is given as $(\mathrm{cm})=$ $176.85 \mathrm{~cm}+0.8 \times[$ (mother's height $(\mathrm{cm})+$ father's height $(\mathrm{cm})) / 2+6.75-176.85]$.

Female adjusted target height is given as $(\mathrm{cm})=$ $163.34 \mathrm{~cm}+0.8 \times[($ mother's height $(\mathrm{cm})+$ father's height $(\mathrm{cm})) / 2-6.75-163.34]$.

With this regression-to-the-mean factor, a patient with tall parents would have an adjusted target height lower than predicted with the traditional midparental height formula. Conversely, a patient with short parents would have an adjusted target height higher than predicted. At the extreme low end of parental heights (e.g., $15 \mathrm{~cm}$ below the mean), this correction would add about $4 \mathrm{~cm}$ to the unadjusted target height. Height and adjusted target height SDS were calculated using reference norms and methodology established by Tanner [6].

Lastly, a corrected height SDS was computed based on an individual's adjusted target height SDS and current height SDS. The corrected height SDS is the current height SDS minus the adjusted target height SDS and provides an estimate of each patient's height deficit within the context of his or her individual genetic growth potential. Severe short stature was defined as a height more than 3 SDs below the mean or 1.5 SDs below the adjusted target height per Growth Hormone Research Society (GRS) guidelines for immediate investigation [7]. Moderate short stature was defined as a height above -3 SDs or less than 1.5 SDs below the adjusted target height.

2.1. Data Analysis. Descriptive statistics are presented as frequency and percentage or mean and standard deviation. All eligible patients are summarized. For selected variables, an additional summary by severity of short stature was generated. Comparisons of auxologic variables by severity of short stature were performed using Student's $t$-tests. Comparisons of frequency of lab tests by severity of short stature were made using Fisher's exact tests. Chi-squared tests were used for comparisons of return rates by severity of short stature. To mitigate the effects of the large sample size, an alpha level of 0.001 was chosen to define statistical significance.

\section{Results}

3.1. Demographics. A total of 21,548 untreated shortstatured patients were eligible for enrollment from 237 participating institutions and clinical practices over the 44month enrollment period. The mean patient age $( \pm S D)$ at enrollment was $8.6 \pm 4.6$ years $(69 \%$ male). The majority of patients were prepubertal $(73 \%)$ and white $(76 \%$ versus $75.1 \%$ of population). The referral rate of African-American (5\% versus $12.3 \%$ of population) and Hispanic (10\% versus $12.5 \%$ of population) patients demonstrated an underrepresentation according to data within the 2000 US Census Bureau [8]. 
The mean $( \pm \mathrm{SD})$ height SDS of referred children was $-2.1( \pm 0.9)$. The mean adjusted target height SDS was -0.3 $( \pm 0.7)$, and the mean corrected height SDS was $-1.8( \pm 1.0)$. The height SDS was below -3 SDs in $13 \%$, between -3 and -2 in $44 \%$, and -2 or above in $44 \%$. In $56 \%$ of patients, heights were more than $1.5 \mathrm{SDs}$ below the adjusted target height. The initial bone age was available in 13,810 patients, and a mean delay of 1.5 years was calculated.

Overall, $59 \%$ of our population met at least one of the GRS criteria for short stature [7] requiring immediate investigation (i.e., height below $-3.0 \mathrm{SDs}$ and/or corrected height SDS more than 1.5 SDs below adjusted target height) (Table 1). Compared with moderately short patients, the severely short patients were slightly older, had a greater bone age delay, and had a higher target height (Table 1).

Turner syndrome was present or diagnosed in 3.4\% of female subjects $(n=226)$. Patients with Turner syndrome with moderate short stature, as compared with those with severe short stature, were younger, had less bone age delay, and had shorter parents (Table 2).

3.2. Screening Tests. Table 3 shows the screening tests reported during the short stature evaluation and the relative frequency of use for moderate and severe short stature. These tests may have been performed prior to referral or at any time during the pediatric endocrinologist's assessment. Bone age and thyroid tests were the most common screening procedures. IGF-1 was reported in $42 \%$ of severely short patients, in $31 \%$ of moderately short patients, and at a similar frequency as the chemistry panel for all patients. Celiac screening was rarely performed. All tests except liver function tests were obtained with a higher frequency in the severely short patients. Although female patients had significantly more karyotypes performed than male patients $(P<.001$ for total group comparison, male data not shown), karyotypes were recorded for only one in five severely short females. As reference, the survey results (percentage of physicians reporting test use during screening) are included. No screening test was recorded at the frequency stated in the survey regardless of the degree of short stature in the patient.

3.3. Return Rate. Although all patients were scheduled for at least one follow-up visit, $38 \%$ of the patients studied did not return after the initial visit and only about 35\% had more than two visits (Figure 1). Among those who failed to return, there was a slight excess of females, patients with moderate short stature, pubertal patients, and AfricanAmericans (Table 4). Auxologic characteristics for patients who returned and those who did not are presented in (Table 5). The most common tests ordered at follow-up visits included bone age (34\%), thyroid panel (22\%), IGF-1 (22\%), and GH stimulation (21\%).

\section{Discussion}

We reviewed data for over 21,000 patients from 237 pediatric endocrine offices who were enrolled in NCGS Substudy 8 to obtain insight into the short stature evaluation. To our

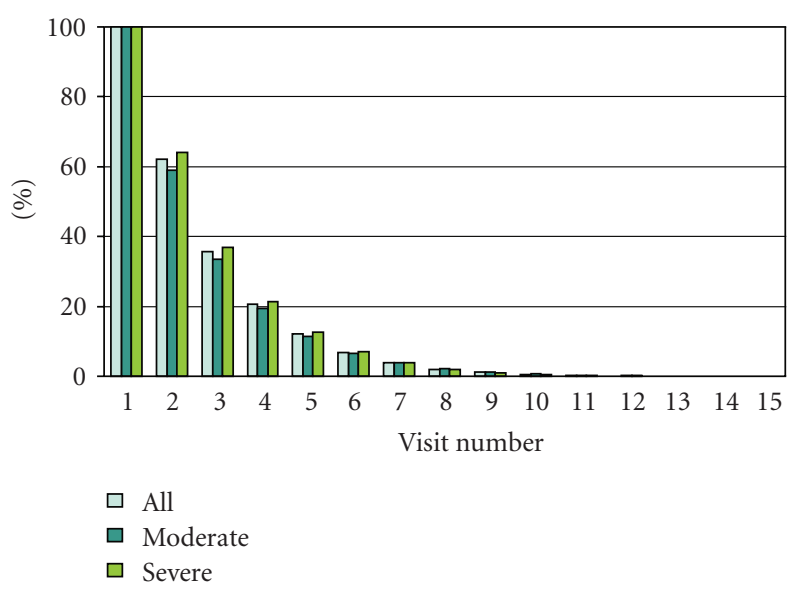

Figure 1: Percentage of patients who had at least " $n$ " visits with a pediatric endocrinologist. For example, about $60 \%$ of all patients had at least 1 follow-up visit (i.e., visit 2).

knowledge, this is the largest published collection of practice data for this type of patients. The size and breadth of this study should provide a good representation of pediatric endocrine practice in the United States. Two findings stand out: the low rate of initial laboratory testing and the low rate of return for scheduled follow-up.

When the reported approach to a short-statured patient (from data obtained in the 1995 survey) and the recorded evaluation in the office setting (data obtained from Substudy 8 ) were compared, large differences between theory and practice were discovered. For example, IGF-1, which may reflect GH adequacy, was obtained in less than $40 \%$ of these short children at the initial visit but was reported as a routine test "always done" in $64 \%$ of patients in the survey. Furthermore, although the comparisons between severely and moderately short patients in (Table 3) are statistically significant, none of the differences in test rates appear to be clinically meaningful; test rates were low essentially regardless of stature.

The reason for the low test rate remains unclear, especially when $59 \%$ of the referred patients met the GRS criteria requiring "immediate investigation." There are some evidence-based guidelines for initial laboratory screening of this population. There may be merit in celiac screening and for karyotyping of short females [9], but there are few data for other tests or for the prevalence of other conditions in short children. Lack of such information limits the scientific validity of most screening guidelines and may be one reason for such a wide variance in usage. Grote et al. have also reported a low degree of laboratory screening in short children referred to either a general clinic or a university clinic [10] in the Netherlands, despite a well-publicized consensus guideline in that country.

Accurate growth records might provide direction to the evaluation [11-13] but may be available for only $50 \%$ of referred children [2]. Forward monitoring may also help direct subsequent laboratory investigation. However, it will be difficult to establish any meaningful growth pattern when 
TABLE 1: Characteristics of all subjects (including subjects with Turner syndrome) by degree of short stature.

\begin{tabular}{llll}
\hline Characteristic & Moderate & Severe & Total \\
\hline Age $(\mathrm{y})^{\mathrm{a}}$ & $8.4( \pm 4.6 ; 8900)$ & $8.8( \pm 4.6 ; 12,648)$ & $8.6( \pm 4.6 ; 21,548)$ \\
Bone age delay $(\mathrm{y})^{\mathrm{a}}$ & $1.1( \pm 1.3 ; 5250)$ & $1.7( \pm 1.4 ; 8615)$ & $1.5( \pm 1.4 ; 13,865)$ \\
Adjusted target height SDS $^{\mathrm{a}}$ & $-0.68( \pm 0.69 ; 7220)$ & $-0.14( \pm 0.63 ; 12,239)$ & $-0.34( \pm 0.7 ; 19,459)$ \\
Height SDS $^{\mathrm{b}}$ & $-1.6( \pm 0.77 ; 8900)$ & $-2.5( \pm 0.86 ; 12,648)$ & $-2.13( \pm 0.94 ; 21,548)$ \\
Corrected height SDS $^{\mathrm{b}}$ & $-0.9( \pm 0.63 ; 7220)$ & $-2.3( \pm 0.76 ; 12,239)$ & $-1.78( \pm 1.01 ; 19,459)$ \\
\hline
\end{tabular}

Values are mean $(\mathrm{SD} ; n)$.

a $P<.001$ moderate versus severe, Student's $t$-test.

b Variables used to define severe or moderate short stature were not compared statistically.

TABLE 2: Characteristics of subjects with Turner syndrome by degree of short stature.

\begin{tabular}{|c|c|c|c|}
\hline Characteristic & Moderate & Severe & Total \\
\hline Age $(y)^{\mathrm{a}}$ & $4.5( \pm 3.7 ; 49)$ & $8.4( \pm 4.7 ; 177)$ & $7.6( \pm 4.8 ; 226)$ \\
\hline Bone age delay $(y)^{\mathrm{a}}$ & $0.3( \pm 0.8 ; 12)$ & $1.4( \pm 1.6 ; 104)$ & $1.3( \pm 1.5 ; 116)$ \\
\hline Adjusted target height SDS ${ }^{\mathrm{a}}$ & $-0.36( \pm 0.71 ; 31)$ & $0.15( \pm 0.71 ; 166)$ & $0.07( \pm 0.73 ; 197)$ \\
\hline Height SDS ${ }^{\mathrm{b}}$ & $-1.51( \pm 0.87 ; 49)$ & $-2.69( \pm 0.89 ; 177)$ & $-2.43( \pm 1.01 ; 226)$ \\
\hline Corrected height SDS ${ }^{\mathrm{b}}$ & $-0.82( \pm 0.77 ; 31)$ & $-2.76( \pm 0.83 ; 166)$ & $-2.46( \pm 1.09 ; 197)$ \\
\hline
\end{tabular}

Values are mean $(\mathrm{SD} ; n)$.

${ }^{a} P<.05$ moderate versus severe, Student's $t$ test.

${ }^{b}$ Variables used to define severe or moderate short stature were not compared statistically.

TABLE 3: Percentage of children for whom screening tests were performed during evaluation.

\begin{tabular}{lccc}
\hline Screening test & $\begin{array}{c}\text { 1995 Survey physicians } \\
\text { (reported) }(n=251) \%\end{array}$ & $\begin{array}{c}\text { Moderate short } \\
\text { stature }(n=8900) \%\end{array}$ & $\begin{array}{c}\text { Severe short } \\
\text { stature }(n=12,648) \%\end{array}$ \\
\hline Bone age & 99 & 59 & 69 \\
Thyroid panel & 97 & 48 & 60 \\
Chemistry panel & 83 & 33 & 39 \\
IGF-1 & 64 & 31 & 42 \\
IGF BP-3 & 28 & 16 & 24 \\
Hematocrit, ESR & 79 & 28 & 32 \\
Karyotype (female) & 69 & 10 & 20 \\
Urinalysis & 79 & 12 & 15 \\
HPG axis evaluation & - & 7 & 6 \\
Celiac screen & 14 & 4 & 6 \\
\hline
\end{tabular}

aReport stated that test was "always used" to screen a short child [3].

${ }^{\mathrm{b}}$ All variables reached a $P<.001$ level of significance comparing severe short stature to moderate short stature by Fishers exact test.

IGF-1:insulin-like growth factor 1; BP:binding protein; ESR:erythrocyte sedimentation rate; HPG:hypothalamic-pituitary-gonad.

TABle 4: Percentage of patients who did not return after initial visit.

\begin{tabular}{llll}
\hline Status & $\%$ & Status & $\%$ \\
\hline All severe short & 36 & All moderate short & 41 \\
All male & 37 & All female & 40 \\
Male severe short & 35 & Male moderate short & 40 \\
Female severe short & 38 & Female moderate short & 43 \\
Male prepubertal & 35 & Male pubertal & 41 \\
Female prepubertal & 37 & Female pubertal & 46 \\
Turner severe short & 38 & Turner moderate short & 43 \\
White males & 35 & African-American males & 44 \\
White females & 38 & African-American females & 46 \\
\hline
\end{tabular}


TABLE 5: Characteristics of male and female subjects by return status.

\begin{tabular}{lllll}
\hline & \multicolumn{2}{c}{ Male } & \multicolumn{2}{c}{ Female } \\
Characteristic & Return & No return & Return & No return \\
\hline Age $(y)$ & $8.9(4.6 ; 9329)$ & $9.1(4.8 ; 5482)$ & $7.5(4.3 ; 4046)^{\mathrm{a}}$ & $8.0(4.5 ; 2691)$ \\
Bone age delay (y) & $1.6(1.3 ; 6283)$ & $1.5(1.3 ; 3445)$ & $1.4(1.4 ; 2503)$ & $1.3(1.5 ; 1634)$ \\
Target height SDS & $-0.32(0.70 ; 8506)$ & $-0.35(0.69 ; 4922)$ & $-0.36(0.70 ; 3628)$ & $-0.38(0.72 ; 2403)$ \\
Height SDS & $-2.11(0.88 ; 9329)^{\mathrm{a}}$ & $-2.04(0.92 ; 5482)$ & $-2.27(0.99 ; 4046)^{\mathrm{a}}$ & $-2.19(1.05 ; 2691)$ \\
Adjusted height SDS & $-1.78(0.95 ; 8506)^{\mathrm{a}}$ & $-1.68(0.99 ; 4922)$ & $-1.91(1.05 ; 3628)^{\mathrm{a}}$ & $-1.80(1.12 ; 2403)$ \\
\hline
\end{tabular}

Values are mean $(\mathrm{SD}, n)$.

${ }^{\mathrm{a}} P<.001$, return status by gender.

almost $40 \%$ of patients will not return after the initial visit and only $35 \%$ will have more than 2 visits regardless of the degree of short stature [14]. Even for those with extended follow-up, a change in height percentiles over time may not be the most effective auxologic screening tool. Distance from adjusted target height (the adjusted height SDS) may be a better tool for guiding laboratory testing [15], but was apparently not often used in this study, as the testing rate was low regardless of the adjusted target height.

The low return rate combined with the low rate of laboratory screening at the initial visit results in an incomplete evaluation in the majority of the referred children. The return rate was poor regardless of the severity of short stature, the sex, or pubertal status, the adjusted target height, or ethnicity. To validate the low return rates, charts of all patients enrolled at one of the authors' sites (the Children's Hospital of Wisconsin) were reviewed in detail. The demographics of this patient subgroup were similar to the national sample. Of the 422 patients enrolled at this site, 135 (32\%) with unexplained short stature did not return after the initial visit. Only one had been treated with growth hormone (and transferred out of the study).

There are several possible interpretations for the poor return rate. Families may not be as concerned about short children as the physicians who refer them or the pediatric endocrinologists who evaluate them. Some may have limited access to specialist care for economic reasons, and some may be influenced by the decision to simply monitor the child. Some patients may have been placed on growth hormone, though this is likely to account for only a small percentage of the low return rate.

The minimal incidence of Turner syndrome in the NCGS Substudy 8 population is $3.4 \%$ ( 226 of 6737 females), about 70 -fold above that in the general population [16]. This reflects the much higher yield when screening short females. As expected, these girls fall farther behind their peers with age. However, a karyotype was done in only $20 \%$ of referred females, despite the common recommendation that short females have such screening $[17,18]$, so we do not know the true incidence of Turner syndrome in this population. The study data do not allow us to determine whether children with Turner syndrome were placed on rhGH treatment, though such treatment may have contributed to the poor recorded return rate.

When one GRS criterion (height SDS less than -3) was applied to our group of patients, only 13\% were classified as severely short. Using another criterion (corrected height SDS below -1.5 ) classified more than half (56\%) of the children as severely short. Thus, two GRS-suggested criteria lead to quite different classifications of the same population. There is some support for preferring the corrected height SDS criteria [15], at least for children above the age of 3 years. Further study is needed to confirm the best auxologic criteria by which one may identify those children most likely to have a pathology associated with their statural deficit.

There are several potential limitations to the study. Participating physicians were responsible for determining which patients needed a follow-up visit and who would be enrolled and for reporting the auxological and laboratory data accurately. The potential for bias in these areas should be somewhat balanced by the breadth of the referral sites and the large number of subjects enrolled. Since the study was closed to data entry about 8 years prior to publication, there may have been some subsequent change in practice patterns.

In summary, we found that children referred to pediatric endocrinologists for short stature evaluation have a low rate of laboratory testing and a low rate of follow-up. Neither finding is related to the severity of statural deficit, whether that deficit is defined relative to peers or parents.

\section{Acknowledgments}

The authors thank the following people from Genentech: Barbara Lippe, M.D., Principal Clinical Scientist for scientific advice and review of the manuscript; Joan Jacobs, M.A., Senior Biostatistician for biostatistical input and review; Joanne Julius Hunold, Biostatistical Consultant for Genentech.

\section{References}

[1] Centers for Disease Control and Prevention Growth Charts, 2000, http://www.cdc.gov/growthcharts/.

[2] A. Grimberg, J. K. Kutikov, and A. J. Cucchiara, "Sex differences in patients referred for evaluation of poor growth," Journal of Pediatrics, vol. 146, no. 2, pp. 212-216, 2005.

[3] D. T. Wyatt, D. Mark, and A. Slyper, "Survey of growth hormone treatment practices by 251 pediatric endocrinologists," Journal of Clinical Endocrinology and Metabolism, vol. 80, no. 11, pp. 3292-3297, 1995. 
[4] D. Wyatt, "Lessons from the national cooperative growth study," European Journal of Endocrinology, vol. 151, no. 1, pp. S55-S59, 2004.

[5] J. M. Tanner, "Auxology," in Diagnosis and Treatment of Endocrine Disorders in Childhood and Adolescence, M. S. Kappy, R. M. Blizzard, and C. J. Migeon, Eds., Thomas, Springfield, Ill, USA, 4th edition, 1994.

[6] J. M. Tanner and P. S. W. Davies, "Clinical longitudinal standards for height and height velocity for North American children," Journal of Pediatrics, vol. 107, no. 3, pp. 317-329, 1985.

[7] Growth Hormone Research Society, "Consensus guidelines for the diagnosis and treatment of growth hormone $(\mathrm{GH})$ deficiency in childhood and adolescence: summary statement of the GH research society," Journal of Clinical Endocrinology and Metabolism, vol. 85, no. 11, pp. 3990-3993, 2000.

[8] F. Hobbs and N. Stoops, Demographic Trends in the 20th Century, Census 2000 Special Reports, Series CENSR-4, U.S. Census Bureau, U.S. Government Printing Office, Washington, DC, USA, 2002.

[9] J. C. W. Van Rijn, F. K. Grote, W. Oostdijk, and J. M. Wit, "Short stature and the probability of coeliac disease, in the absence of gastrointestinal symptoms," Archives of Disease in Childhood, vol. 89, no. 9, pp. 882-884, 2004.

[10] F. K. Grote, W. Oostdijk, S. M. P. F. De Muinck KeizerSchrama, et al., "The diagnostic work up of growth failure in secondary health care: an evaluation of consensus guidelines," BMC Pediatrics, vol. 8, pp. 21-29, 2008.

[11] J. M. Himes, "Minimum time intervals for serial measurements of growth in recumbent length or stature of individual children," Acta Paediatrica, vol. 88, no. 2, pp. 120-125, 1999.

[12] American Academy of Pediatrics Committee on Drugs and Committee on Bioethics, "Considerations related to the use of recombinant human growth hormone in children," Pediatrics, vol. 99, no. 1, pp. 122-129, 1997.

[13] D. S. Hardin, J. Woo, R. Butsch, and B. Huett, "Current prescribing practices and opinions about growth hormone therapy: results of a nationwide survey of paediatric endocrinologists," Clinical Endocrinology, vol. 66, no. 1, pp. 85-94, 2007.

[14] W. A. Marshall, "Evaluation of growth rate in height over periods of less than one year," Archives of Disease in Childhood, vol. 46, no. 248, pp. 414-420, 1971.

[15] F. K. Grote, P. Van Dommelen, W. Oostdijk, et al., "Developing evidence-based guidelines for referral for short stature," Archives of Disease in Childhood, vol. 93, no. 3, pp. 212-217, 2008.

[16] J. Nielsen and M. Wohlert, "Chromosome abnormalities found among 34910 newborn children: results from a 13-year incidence study in Arhus, Denmark," Human Genetics, vol. 87, no. 1, pp. 81-83, 1991.

[17] R. G. Rosenfeld, L.-G. Tesch, L. J. Rodriguez-Rigau, et al., "Recommendations for diagnosis, treatment, and management of individuals with Turner syndrome," Endocrinologist, vol. 4, no. 5, pp. 351-358, 1994.

[18] P. Saenger, K. A. Wikland, G. S. Conway, et al., "Recommendations for the diagnosis and management of Turner syndrome," Journal of Clinical Endocrinology and Metabolism, vol. 86, no. 7, pp. 3061-3069, 2001. 